\title{
Miniature Language Acquisition Tasks Using Dynamic Weightless Systems
}

\author{
Nicholas J. Sales
}

Neural Systems Engineering Research Group, Department of Electrical and Electronic Engineering, Imperial College of Science, Technology and Medicine, London SW7 2BT.

email: cyber@ee.ic.ac.uk

\begin{abstract}
The GNU weightless Artificial Neural Network (ANN) [2] has properties which are important for the modelling of language acquisition in humans. One property, described in this paper, is the ability to successfully learn associations between symbols that have been embedded in large amounts (up to 85\%) of random noise.

The "Symbol Grounding Problem" has figured large in language acquisition research for many years, and it was noted by Harnad [3], that connectionist methods might be employed to make feasible a solution to this problem.

Work is under way to model acquisition of perceptually grounded symbols, and folk physics, (common sense interaction with the physical world), using dynamic recurrent weightless systems [6]. This paper examines a simple example of acquisition tasks using weightless systems.

The system whose behaviour is investigated here is a GNU (General Neural Unit), constructed using multiple GRAM's (Generalising RAM's) [1]. There are many results for this system, e.g., Ntourntoufis' work, [4], but the behaviour of the system in language acquisition tasks has not been investigated before. The properties investigated here are directly relevant to our approach to the study of language acquisition (outlined in [5]).

Experiments were carried out using simulated recurrent GNU's, composed of between 4 and 8 GRAM's. Simple 4 and 8 step sequences were easily learned by the networks. The sequences were then interspersed, first with noisy versions of themselves, then with randomly generated noise. The networks still learned the sequences with $100 \%$ accuracy, even when correct sequences composed only $15 \%$ of the total training inputs. Individual steps in the sequences were then dispersed through a long sequence of random noise, but the networks still succeeded in learning the sequences with $100 \%$ accuracy, with only about $10 \%$ of the training inputs being relevant.

Such properties are important to our model of language acquisition, because they are exactly the kind of learning processes one could expect children to go through, when first learning the simple connections between nouns and referents. Many parts of linguistic input are essentially ignored or incompletely understood, but the child understands that whenever "horse" is used indicatively, there will be a horse present.
\end{abstract}

\section{REFERENCES}

1. Aleksander, I., "Ideal Neurons for Neural Computers", in Parallel Processing in Neural Systems and Computers 1990, pp. 225-228, Elsevier Science Publishers, Amsterdam, 1990.

2. Aleksander, I., "Neural Systems Engineering: Towards a Unified Design Discipline", Computing \& Control Engineering Journal, pp. 259-265, November 1990.

3. Harnad, S., "The Symbol Grounding Problem", Physica D, vol. 42, pp. 335-346, 1990.

4. Ntourntoufis, P., "Capacity and Retrieval Properties of an Auto-associative G.N.U”, (Abstract only) in Proceedings of IJCNN '91 Seattle, IEEE, New York., 1991.

5. Sales, N. J., "The Essentials Of Language Acquisition: What Do We Need To Study", NSEIR/NJS\#2/92, Neural Systems Engineering Research Group Internal Report, London, December 1992.

6. Sales, N. J., "Motivating The Use Of Neural Systems For Language Acquisition: Can Grounding Of Symbols And Development Of Folk Physics Provide A Framework For Language", NSEIR/NJS\#3/92, Neural Systems Engineering Research Group Internal Report, London, December 1992. 